\title{
UNDERSTANDING THE EFFECTS OF STRESS ON THE COEFFICIENT OF THERMAL EXPANSION
}

\author{
Quang X. Le ${ }^{1,3}$, Jose L. Torero², Vinh TN Dao ${ }^{1^{*}}$ \\ ${ }^{1}$ School of Civil Engineering, The University of Queensland, Brisbane, Australia QLD 4072 \\ 2 Department of Fire Protection Engineering, the University of Maryland 20740 \\ ${ }^{3}$ Faculty of Civil Engineering, The University of Danang - University of Science and Technology, \\ Danang, Vietnam
}

\section{Abstract}

Important coupled effects of stress and temperature change have long been recognised and taken into account in models assessing the performance of solid materials. However, due to incomplete understanding, existing models used for concrete are essentially empirical and lack rational basis. This limits their predictive capability and applicability range. In this paper, fundamental thermodynamics and continuum mechanics laws are used to obtain an expression quantitatively predicts the effects of stress on the coefficient of thermal expansion. The newly-defined thermal expansion coefficient is then used to develop a rational model, which is shown to be capable of reliably quantifying the coupling effects of stress and temperature change. Good agreement between the model predictions, available data and observations is obtained. Equally important, all parameters of the developed model can be determined using simple tests. This enables a wide adoption of the model in practical engineering applications. While this paper uses data for concrete, the proposed model is developed from first principles and using fundamental physical laws, therefore, it should be applicable to a wide range of solid materials.

\section{Introduction}

Structures are commonly exposed to rapid temperature changes due to fire, climatic conditions, radiation or heat developed from industrial processes. The combined effects of mechanical and thermal loading can give rise to significant level of stress and damage as a consequence of nonlinear temperature and strain profiles and of restrained structural deformation. Indeed, the coupling effects between stress and temperature increase have been widely observed experimentally and the critical need to properly account for these effects in assessing the performance of solid materials has been broadly acknowledged ${ }^{1-9}$. Such proper assessment is particularly important if a certain level of performance is strictly required in case of accidental loading: Typical examples include (i) nuclear reactors overheated due to a failure of the cooling system (e.g. in the recent Fukushima accident) and (ii) columns and shear walls in high-rise buildings in case of fires.

Unfortunately, the coupling effects between stress and temperature increase are currently mainly accounted for in structural analysis and design through the introduction of empirical correlations $s^{1,2,7,10-14}$. As these empirical correlations are principally developed by best-fitting to limited test data, their applicability to scenarios different from those tested remains questionable and their reliability hinges on the quality and extent of the corresponding test data. 
As a result, more rational models developed from first principles and based on fundamental physical laws are required. Additionally, in order for these models to be widely adopted in engineering applications, besides being adequately robust, they need to be sufficiently simple and their parameters are required to be conveniently determined using widely-available test setups and procedures.

This paper presents one such rational model for solid materials subject to combined effects of stress and temperature increase, developed using fundamental laws of thermodynamics and continuum mechanics. Evidence for the predictive capability of the proposed firstprinciples model will be highlighted, together with a simple methodology to conveniently determine the model parameters.

\section{Nomenclature}

\begin{tabular}{|c|c|c|c|}
\hline$E$ & $=$ Young's modulus & $\sigma$ & $=$ uniaxial stress \\
\hline$E_{0}$ & $=$ Young's modulus at $T_{a m b}$ & $\sigma_{u T}$ & compressive strength at $\mathrm{T}$ \\
\hline$E(T)$ & Young's modulus at $T$ & $\sigma_{u 0}$ & $=$ compressive strength at $T_{a m b}$ \\
\hline$E_{\text {eff }}(T)$ & effective Young's modulus at $T$ & $\sigma_{0}$ & $=$ compressive stress at $T_{a m b}$ \\
\hline$\varepsilon_{i j}, \varepsilon_{t o t}$ & total strain & $\sigma(T)$ & $=$ thermal stress at $T$ \\
\hline$\varepsilon$ & uniaxial strain & $\sigma_{i j}$ & $=$ stress component \\
\hline$\varepsilon_{\sigma}(T)$ & mechanical strain at $T$ & $U$ & $=$ internal energy per unit mass \\
\hline$\varepsilon_{t h}(T)$ & $=$ free thermal expansion strain at $T$ & $R$ & $=$ internal heat produced per unit \\
\hline$\varepsilon_{\text {lits }}(T)$ & load-induced thermal strain at $T$ & & mass \\
\hline$T_{a m b}$ & $=$ ambient temperature & $Q$ & $=$ heat loss \\
\hline$T$ & $=$ temperature & $A$ & $=$ Helmholtz free energy function \\
\hline$\Delta T$ & $=T-T_{a m b}$ & $S$ & $=$ specific entropy \\
\hline$\alpha_{0}$ & free thermal expansion coefficient & $\rho_{0}$ & $=$ density \\
\hline$\alpha$ & $=$ thermal expansion coefficient & $c_{e}$ & $=$ specific heat \\
\hline$\alpha_{\sigma}$ & $\begin{aligned}= & \text { stress-dependent thermal expansion } \\
& \text { coefficient }\end{aligned}$ & & \\
\hline
\end{tabular}

\section{Background}

Theoretically, the total strain of solid materials in simultaneous load and heat conditions has been traditionally described by using Duhamel-Neumann constitutive law. The general model of Duhamel-Neumann model ${ }^{15-17}$ is as follows:

$$
\varepsilon_{i j}=\frac{1}{E}\left[(1+v) \sigma_{i j}-v \cdot \delta_{i j} . \sigma_{k k}\right]+\alpha \cdot \delta_{i j} \Delta T
$$

where: $\varepsilon_{i j}$ is principal strain for $i=j$ and shear strain for $i \neq j ; \sigma_{i j}$ is principal stress for $i=j$ and shear stress for $i \neq j ; \delta_{i j}$ is the Kronecker delta being one for $i=j$ and zero otherwise; $\sigma_{k k}$ equals the summation of principal stresses $\sigma_{11}+\sigma_{22}+\sigma_{33}$. Poison's ratio is $v, \alpha$ is the thermal expansion coefficient and $\Delta T$ is the temperature above that of ambient.

While analyzing structural elements under uniaxial loading and elevated temperatures, the following simplifications are possible:

$$
\sigma_{11}=\sigma ; \sigma_{22}=\sigma_{33}=0
$$

The constitutive law of linear thermo-elasticity can then be written as: 


$$
\varepsilon_{t o t}=\varepsilon_{\sigma}(T)+\varepsilon_{t h}(T)=\frac{\sigma}{E}+\alpha . \Delta T
$$

where, $\sigma$ is the stress and $E$ is Young's modulus.

When subjected to increasing temperature, thermal loads can induce restraint stresses if the elements are not free to expand. The resulted stresses induced by load and restraint thermal deformation in turn will affect the thermal deformation of structural elements ${ }^{18}$. The above coupling effects need to be properly accounted for in such rational constitutive model as the Duhamel-Neumann law ${ }^{19}$, which in turn requires clear understanding of each component of the model. While the mechanical part of Duhamel-Neumann model has been clearly defined, it is however still unclear whether the thermal expansion coefficient $(\alpha)$ is:

(i) Free thermal expansion coefficient determined in the condition of zero stress $\left(\alpha_{0}\right)^{15,16}$; or

(ii) Stress-dependent thermal expansion coefficient $\left(\alpha_{\sigma}\right)^{18,20}$ : In case where $\alpha$ is assumed as $\alpha_{\sigma}$, the formula for $\alpha_{\sigma}$ remains unclearly defined ${ }^{18}$ or inaccurately derived $^{20}$.

Due to such incomplete understanding, the coupling effects between stress and temperature increase have been predominantly taken into account in structural analysis and design through the introduction of empirical correlations ${ }^{1,2,7,10-14,21}$. The prevalent use of empirical correlations is clearly demonstrated in the subsequent section through the case of concrete, the most commonly used construction material, at elevated temperatures. A similar approach of empirical fitting to the test data could be found in case of steel when investigating mechanical responses at elevated temperatures under heat and load conditions ${ }^{21-23}$.

\section{Concrete and its load-induced thermal strain at elevated temperatures}

Tests conducted on concrete subject to various stress-thermal loading paths have experimentally shown that the total thermal strain of concrete ( $\varepsilon_{\text {tot }}$ ) at elevated temperatures is significantly reduced if the concrete is heated when loaded ${ }^{10,12,24}$. Similarly, the recorded mechanical reaction force of restrained samples while heating is drastically less than those calculated using Duhamel-Neumann model if the free thermal expansion coefficient $\left(\alpha_{0}\right)$ is used.

In a prior study conducted by Anderberg and Thelandersson ${ }^{10}$, concrete samples were first loaded at ambient temperature and then heated to target temperature levels. It was observed that under a constant compressive stress of $22.5 \%$ of the ambient compressive strength, the total strain was between $55 \%$ and $70 \%$ smaller compared to that of the comparable samples heated in a zero-stress condition. This reduction is even higher if the sample is subjected to higher compressive stress levels before heating.

Understanding and incorporating such reduction of strain in the constitutive model is vital to assess and simulate the performance of concrete structures in fire conditions. Therefore, 
correction values for the strain component have been introduced. These corrections are described as load-induced thermal strain (LITS) and creep strain, and have been added into the Duhamel-Neumann constitutive model since the $1970 \mathrm{~s}^{10}$. The total strain of loaded concrete samples in heating and loading conditions has therefore been mathematically modified in a manner that is described by a simple summation of effects as per Equation $(4)^{25}$ :

$$
\varepsilon_{t o t}=\varepsilon_{t h}(T)+\varepsilon_{\sigma}(T)+\varepsilon_{\text {lits }}(T, \sigma)+\varepsilon_{c r}(T)
$$

where: $\varepsilon_{\text {tot }}$ is the total strain; $\varepsilon_{\text {th }}$ is free thermal strain; $\varepsilon_{\sigma}$ is the mechanical strain caused by corresponding stress $\sigma$, Elits is the transient thermal strain or load-induced thermal strain; and $\varepsilon_{\mathrm{cr}}$ is the creep strain.

During rapid temperature increase, the thermal creep strain is typically negligibly small compared to the total strain ${ }^{10}$. In Anderberg and Thelandersson ${ }^{10}$, for instance, the maximum creep strain $\left(\varepsilon_{c r}(T)\right)$ over a 3 -hour period was found less than $5 \%$ of the corresponding total strain. Therefore, Equation (4) can be revised to:

$$
\varepsilon_{\text {tot }}=\varepsilon_{\text {th }}(T)+\varepsilon_{\sigma}(T)+\varepsilon_{\text {lits }}(T, \sigma)
$$

Since 2004, the LITS or transient creep strain or transient thermal strain has long been implicitly included in the stress-strain constitutive model of concrete at elevated temperatures in Eurocode Standard ${ }^{25,26}$. Strength and deformation of concrete at elevated temperatures are then suggested to be obtained from this stress-strain relationship. In addition, it is also suggested that the thermally-induced strains and stress both due to temperature changes into the mechanical response need to be considered by using the relation as shown in the Equation $(5)^{25}$. However, the design value of the LITS is not explicitly derived or nominated, thus the model of $\varepsilon_{\text {lits }}$ remains unclear in the design model in Eurocode Standard.

Many correlations for $\varepsilon_{\text {lits }}$ have been developed since the 1970's, mainly on the basis of available experimental data ${ }^{10,12,13,27}$. To calculate $\varepsilon_{\text {lits, }}$ the following approach has been used:

$$
\varepsilon_{\text {lits }}(T, \sigma)=\varepsilon_{t o t}-\varepsilon_{t h}(T)-\varepsilon_{\sigma}(T)
$$

where, $\varepsilon_{t o t}$ is the measured total strain of concrete under load and heat condition; $\varepsilon_{t h}$ is free thermal strain, recorded from un restrained concrete sample heated without applied stress; and $\varepsilon_{\sigma}$ is mechanical strain, calculated by dividing mechanical forces by the instantaneous Young's modulus.

The correlations describing $\varepsilon_{\text {lits }}$ are sometimes expressed as functions of temperature and the ratio between applied stress $(\sigma)$ and compressive strength of the concrete at ambient temperature $\left(\sigma_{u 0}\right)$. As an example, Anderberg and Thelandersson 10 used their experimental data to develop a mathematical correlation where $\varepsilon_{\text {lits }}$ is represented as a linear function of free thermal strain $\left(\varepsilon_{t h}\right)$ by introducing a correction factor $k$, as follows:

$$
\varepsilon_{\text {lits }}(T, \sigma)=k \cdot \frac{\sigma}{\sigma_{u 0}} \cdot \varepsilon_{t h}
$$


The value of $k$ was calculated by using a linear regression analysis that compared the value of $\varepsilon_{\text {lits }} /\left(\sigma / \sigma_{u 0}\right)$ against the value of free thermal expansion strain $\left(\varepsilon_{t h}\right)$. This regression analysis gave values of the correction factor $(k)$ ranging from 1.8 to 2.35 . The fit delivered good agreement between measured and calculated strains in the range of temperatures between ambient and $500^{\circ} \mathrm{C}$, as shown in Figure 1. It should be noted that Anderberg and Thelandersson ${ }^{10}$ failed to clearly define what fundamental physical behavior they were attempting to include in their correlation. Also, their correlation is sensitive to the value of $k$ resulting in values for the load-induced thermal strain that changes within a range of approximately $\pm 30 \%$ (Figure 1 ).

Other corrections have been introduced to account for the non-linearity nature of the data with temperature. Diederichs ${ }^{28}$ developed a LITS correlation by using a third order polynomial of the temperature to obtain a best fit to experimental data (cited by Li and Purkiss ${ }^{29}$, and Torelli, et al. $\left.{ }^{1}\right)$. Thus the ratio between $\varepsilon_{\text {lits }} /\left(\sigma / \sigma_{u 0}\right)$ is defined as a polynomial function of temperature (Equation (8)) and is also plotted in Figure 1.

$$
\begin{aligned}
\varepsilon_{\text {lits }}(T, \sigma)= & \left(4.12 \times 10^{-5}(T-20)-1.72 \times 10^{-7}(T-20)^{2}\right. \\
& \left.+3.3 \times 10^{-10}(T-20)^{3}\right) \cdot \frac{\sigma}{\sigma_{u 0}}
\end{aligned}
$$

Although the Diederichs's correlation allows a better fit to the data, it still fails to explain the fundamental physical basis to the polynomial fit. Also, the value of the LITS is sensitive to the parameters used for the polynomial function. These parameters are a function of the concrete type, thus can only be used to quantify materials consistent with that of the test.

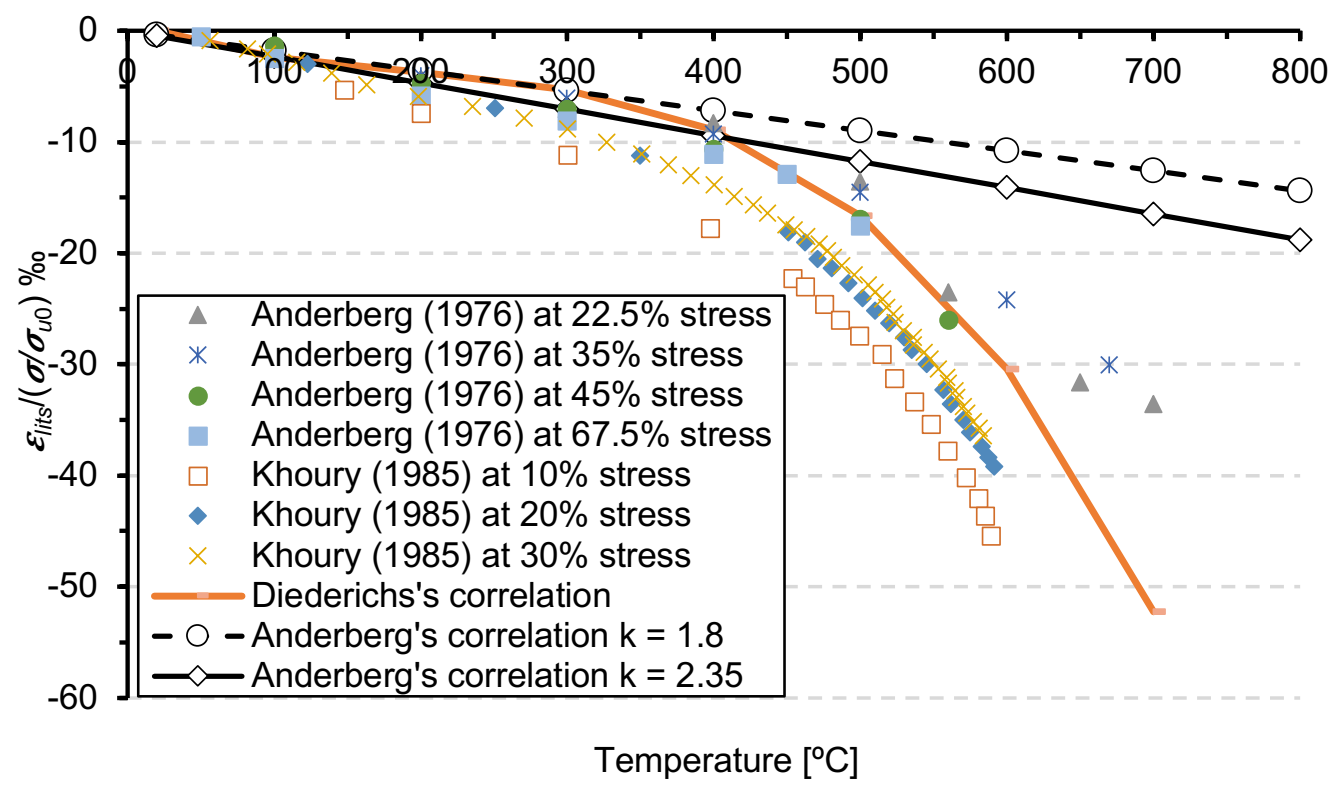

Figure 1. Plot the ratio of $\varepsilon_{\text {lits }} /\left(\sigma / \sigma_{u 0}\right)$ against the temperature of Anderberg and Thelandersson ${ }^{10}$, Khoury, et al. ${ }^{30}$ compared to Diederichs ${ }^{28}$ and Anderberg and Thelandersson ${ }^{10}$ fitting correlations.

Gernay and Franssen ${ }^{31}$ proposed a correlation of transient thermal strain or LITS using the stress-strain constitutive models from ENV 1992-1-2:1995 ${ }^{32}$ and EC2 1992-1-2:2004 ${ }^{25}$ together with the test data conducted by Schneider ${ }^{33}$, as Equation (9): 


$$
\varepsilon_{\text {lits }}(T, \sigma)=\varnothing(T) \cdot \frac{\sigma}{\sigma_{u 0}}=\frac{2}{3} \frac{\left(\varepsilon_{c 1, E C 2}-\varepsilon_{c 1, \text { min }}\right)}{\sigma_{u T} / \sigma_{u 0}} \frac{\sigma}{\sigma_{u 0}}
$$

where, $\varnothing(T)$ is a nonlinear function of temperature; $\varepsilon_{c 1, E C 2}$ is the peak-stress strain in EN 1992-1-2:2004; $\varepsilon_{c 1, \min }$ is the peak-stress strain in ENV 1992-1-2:1995. The significance of this correlation is to model the irreversibility of transient creep strain, thus allows better modelling the performance of concrete structures in both heating and cooling phases ${ }^{34}$. However, the fundamental physic of the load-induced thermal strain or transient thermal strain has not been clearly incorporated into this correlation.

Subsequent correlations attempted to generalize the LITS functions to include not only temperature but also the concrete type and the magnitude of the load imposed. In the 1980s, Khoury, et al. ${ }^{12}$ reported further experimental data and developed "master-curves" for different aggregates types and concretes, including limestone aggregate, gravel aggregate, basalt concrete, and lightweight concrete (Figure 1). The applicability of these "master-curves" to the wide range of concretes currently commercially available, however, remains to be ascertained.

Terro ${ }^{13}$ re-analysed the data of Khoury, et al. ${ }^{12}$ and substituted the "master curves" by Equation (13). The author considered LITS as a linear function of the applied stress and a polynomial function of the temperature. It should be noted that Terro ${ }^{13}$ defined the LITS as the combination of transient thermal strain and mechanical strain in his model. Equation (10) presents the LITS for lightweight concrete containing $65 \%$ of calcareous aggregate and loaded before heating at $30 \%$ of compressive strength at ambient:

$$
\begin{aligned}
\varepsilon_{\text {lits }}\left(T, 0.3 \sigma_{u 0}\right) & \left.\right|_{65 \%} \\
& =\left(43.87-2.73 T-6.35 \times 10^{-2} T^{2}+2.19 \times 10^{-4} T^{3}\right. \\
& \left.-2.77 \times 10^{-7} T^{4}\right) \times 10^{-6}
\end{aligned}
$$

in a similar manner Equation (11) shows the LITS for gravel aggregate concrete

$$
\begin{aligned}
\varepsilon_{\text {lits }}\left(T, 0.3 \sigma_{u 0}\right) & \left.\right|_{65 \%} \\
& =1.48 \times 10^{-6}\left(1098.5-39.21 T+0.43 T^{2}\right) \\
& -1.48 \times 10^{-9}\left(2.44 T^{3}-6.27 \times 10^{-3} T^{4}\right. \\
& \left.+5.95 \times 10^{-6} T^{5}\right)
\end{aligned}
$$

For different stress levels, the LITS is calculated using a linear correction to the $30 \%$ load LITS as presented in Equation (12):

$$
\left.\varepsilon_{\text {lits }}(T, \sigma)\right|_{65 \%}=\left.\left(0.032+3.226 . \frac{\sigma}{\sigma_{u 0}}\right) \varepsilon_{\text {lits }}\left(T, 0.3 \sigma_{u 0}\right)\right|_{65 \%}
$$

In an attempt to solve the mentioned weakness of the "master-curves" in Khoury's model, Terro ${ }^{13}$ introduced the correction factor $V_{a}$, which is the volume fraction of aggregate in concrete samples. The general LITS model developed is then:

$$
\varepsilon_{\text {lits }}(T, \sigma)=\left.\frac{V_{a}}{0.65} \varepsilon_{\text {lits }}(T, \sigma)\right|_{65 \%}
$$


It should be emphasized that this model was developed using the "master-curves", which contained a higher level of deviation at high temperatures. Also, similar to those proposed by Anderberg and Thelandersson ${ }^{10}$, LITS correlations suggested by Terro ${ }^{13}$ lack rational links to the fundamental physical basis and are also highly sensitive to the different fitting parameters.

Alternative functions have also been reported but in most cases simply introduced different fits to the same data. For example, Nielsen, et al. ${ }^{27}$ developed a similar model to that of Anderberg and Thelandersson ${ }^{10}$ which described the LITS as a linear function of temperature and the ratio between applied stress $(\sigma)$ against ambient compressive strength $\left(\sigma_{u 0}\right)$ :

$$
\varepsilon_{\text {lits }}(T, \sigma)=b . T \cdot \frac{\sigma}{\sigma_{u 0}}
$$

where, $b=38 \times 10^{-6}$. The value of " $b$ " was calibrated to obtain the best fit with Terro's model and the "master-curves" developed by Khoury, et al. ${ }^{12}$. No attempt was made to quantify the higher deviation of the "master curves" at high temperature and to quantify the association between material properties and LITS. Table 1 presents a summary of the above-discussed models.

Table 1. Load-induced thermal strain correlations developed by different authors.

\begin{tabular}{|c|c|c|}
\hline Study & LITS correlation & Notes \\
\hline $\begin{array}{l}\text { Anderberg } \\
\text { and } \\
\text { Thelandersson } \\
10\end{array}$ & $\varepsilon_{\text {lits }}(T, \sigma)=k \cdot \frac{\sigma}{\sigma_{u 0}} \cdot \varepsilon_{t h}$ & $20-500{ }^{\circ} \mathrm{C}$ \\
\hline Terro ${ }^{13}$ & 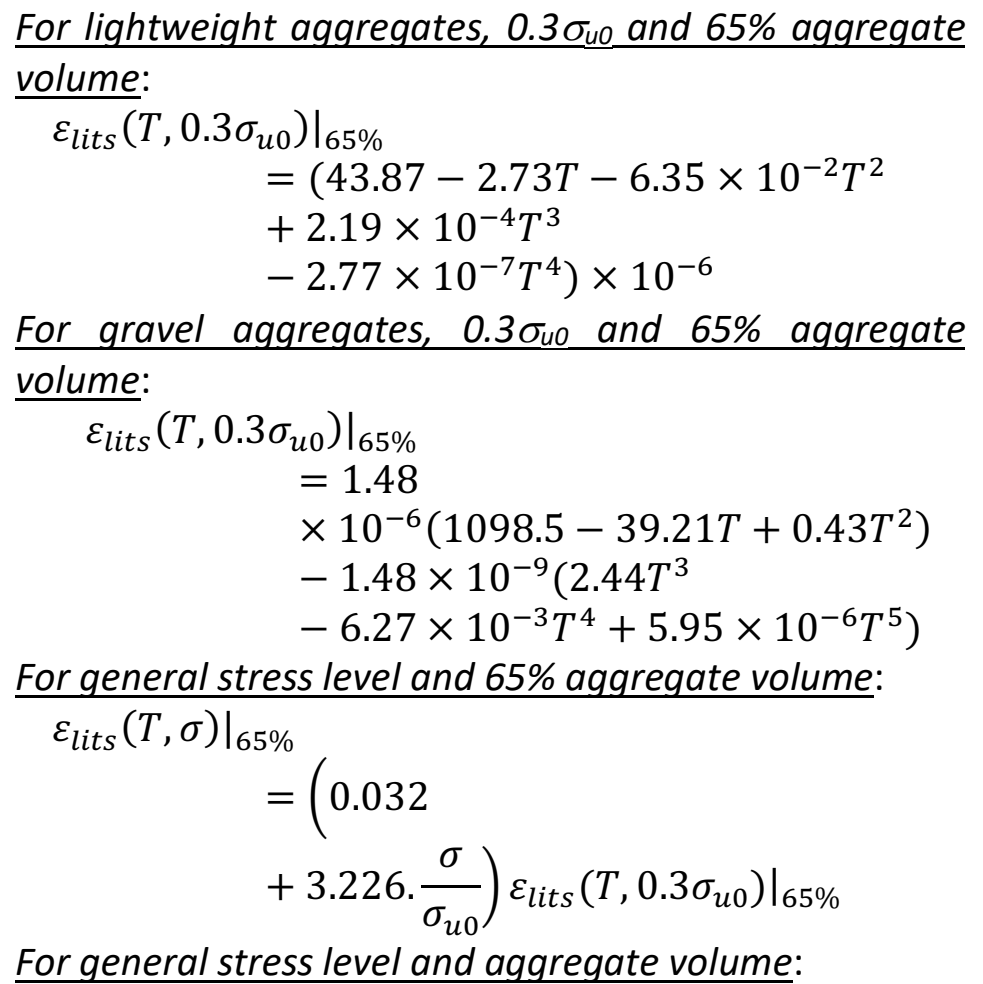 & $\begin{array}{l}20-600{ }^{\circ} \mathrm{C} \\
0.3 \sigma_{u} 0 \text { : } \\
\text { Stress level } \\
\text { of } 30 \% \text { of } \\
\text { ambient } \\
\text { compressive } \\
\text { strength } \\
\sigma_{\mathrm{u} 0} \text {; } \\
\mathrm{V}_{\mathrm{a}} \text { : Volume } \\
\text { fraction of } \\
\text { aggregate in } \\
\text { concrete }\end{array}$ \\
\hline
\end{tabular}




\begin{tabular}{|c|c|c|}
\hline & $\varepsilon_{\text {lits }}(T, \sigma)=\left.\frac{V_{a}}{0.65} \varepsilon_{\text {lits }}(T, \sigma)\right|_{65 \%}$ & \\
\hline $\begin{array}{l}\text { Nielsen, et al. } \\
27\end{array}$ & $\varepsilon_{\text {lits }}(T, \sigma)=b . T \cdot \frac{\sigma}{\sigma_{u 0}}$ & $\begin{array}{l}20-600^{\circ} \mathrm{C} \\
b=38 \times 10^{-6}\end{array}$ \\
\hline $\begin{array}{l}\text { Gernay and } \\
\text { Franssen }{ }^{31}\end{array}$ & $\varepsilon_{\text {lits }}(T, \sigma)=\emptyset(T) \cdot \frac{\sigma}{\sigma_{u 0}}=\frac{2}{3} \frac{\left(\varepsilon_{c 1, E C 2}-\varepsilon_{c 1, \min }\right)}{\sigma_{u T} / \sigma_{u 0}} \frac{\sigma}{\sigma_{u 0}}$ & $20-800^{\circ} \mathrm{C}$ \\
\hline Diederichs ${ }^{28}$ & $\begin{aligned} \varepsilon_{\text {lits }}(T, \sigma)= & \left(4.12 \times 10^{-5}(T-20)\right. \\
& -1.72 \times 10^{-7}(T-20)^{2} \\
& \left.+3.3 \times 10^{-10}(T-20)^{3}\right) \cdot \frac{\sigma}{\sigma_{u 0}}\end{aligned}$ & $20-800^{\circ} \mathrm{C}$ \\
\hline
\end{tabular}

In the ensuing section, a more rational model accounting for the coupling effects between stress and temperature increase is derived on the basis of fundamental laws of thermodynamics and continuum mechanics. The predictive capability of the newly-derived model is then demonstrated through comparing its predictions with corresponding experimental data and observations.

\section{Theoretical analysis}

Assuming no temperature gradient within the sample, the first law of thermodynamics results in Equation (15) as the description of conservation of energy:

$$
\rho_{0}\left(\frac{\partial U}{\partial t}-\frac{\partial R}{\partial t}\right)=\sigma \cdot \frac{\partial \varepsilon}{\partial t}-Q
$$

where: $U$ is the internal energy per unit mass; $R$ is the internal heat produced per unit mass; $\rho_{0}$ is the density of solid; $\sigma$ and $\varepsilon$ are stress and strain of solid in uniaxial loading condition; and $Q$ is the total heat loss.

Assuming no heat production and negligible heat losses, the conservation of energy equation can be further simplified to:

$$
\rho_{0} \frac{\partial U}{\partial t}=\sigma \cdot \frac{\partial \varepsilon}{\partial t}
$$

The Helmholtz free energy function $A$ can be used to describe the relation of the internal energy $U$, temperature $T$, and the entropy $S$, as follows:

$$
A=U-T S
$$

By differentiating the Helmholtz free energy respect to time, we get:

$$
\frac{\partial A}{\partial t}=\frac{\partial U}{\partial t}-S \cdot \frac{\partial T}{\partial t}-T \cdot \frac{\partial S}{\partial t}
$$

Given that for elastic materials, the Helmholtz energy $A$ is a function of strain $(\varepsilon)$ and temperature $(T)$. Then, the rate of change of $A$ can be determined by using the chain rule:

$$
\frac{\partial A}{\partial t}=\frac{\partial A}{\partial \varepsilon} \cdot \frac{\partial \varepsilon}{\partial t}+\frac{\partial A}{\partial T} \frac{\partial T}{\partial t}
$$

Substituting Equation (19) into (18) gives: 


$$
\frac{\partial U}{\partial t}=\frac{\partial A}{\partial \varepsilon} \cdot \frac{\partial \varepsilon}{\partial t}+\frac{\partial A}{\partial T} \frac{\partial T}{\partial t}+S \cdot \frac{\partial T}{\partial t}+T \cdot \frac{\partial S}{\partial t}
$$

Equation (20) can be then substituted into (16), resulting in:

$$
\left(\rho_{0} \frac{\partial A}{\partial \varepsilon}-\sigma\right) \frac{\partial \varepsilon}{\partial t}+\rho_{0}\left(\frac{\partial A}{\partial T}+S\right) \frac{\partial T}{\partial t}+\rho_{0} T \cdot \frac{\partial S}{\partial t}=0
$$

With the assumption of negligible heat produced and negligible heat losses, the second law of thermodynamics requires:

$$
\rho_{0} T \cdot \frac{\partial S}{\partial t}=0 ; \quad \text { leading to: } \quad \frac{\partial S}{\partial t}=0
$$

As $\partial \varepsilon / \partial t$ and $\partial T / \partial t$ are arbitrary, the non-trivial solution of Equation (21) leads to:

$$
\begin{aligned}
& \sigma=\rho_{0} \frac{\partial A}{\partial \varepsilon} \\
& S=-\frac{\partial A}{\partial T}
\end{aligned}
$$

Substituting Equation (24) into Equation (22) and applying the chain rule, we obtain:

$$
\begin{aligned}
\frac{\partial S}{\partial t}= & -\frac{\partial}{\partial t}\left(\frac{\partial A}{\partial T}\right)=-\frac{\partial}{\partial T}\left(\frac{\partial A}{\partial t}\right)=-\frac{\partial}{\partial T}\left(\frac{\partial A}{\partial \varepsilon} \cdot \frac{\partial \varepsilon}{\partial t}+\frac{\partial A}{\partial T} \frac{\partial T}{\partial t}\right) \\
& =-\frac{\partial^{2} A}{\partial \varepsilon \partial T} \cdot \frac{\partial \varepsilon}{\partial t}-\frac{\partial A}{\partial \varepsilon} \frac{\partial^{2} \varepsilon}{\partial T \partial t}-\frac{\partial^{2} A}{\partial T^{2}} \frac{\partial T}{\partial t}-\frac{\partial A}{\partial T} \frac{\partial^{2} T}{\partial T \partial t}
\end{aligned}
$$

Since $\partial^{2} \varepsilon / \partial T \partial t=0$, and $\partial^{2} T / \partial T \partial t=0$, Equation (25) can be rewritten as:

$$
\frac{\partial S}{\partial t}=-\frac{\partial^{2} A}{\partial \varepsilon \partial T} \cdot \frac{\partial \varepsilon}{\partial t}-\frac{\partial^{2} A}{\partial T^{2}} \frac{\partial T}{\partial t}
$$

Differentiating the stress in Equation (23) respect the temperature T gives:

$$
\frac{\partial \sigma}{\partial T}=\rho_{0} \cdot \frac{\partial^{2} A}{\partial \varepsilon \partial T}
$$

Substituting Equation (27) into Equation (26) and multiplying both sides of the resulting equation with $T$, we obtain:

$$
\rho_{0} T \frac{\partial S}{\partial t}=-T \cdot \frac{\partial \sigma}{\partial T} \cdot \frac{\partial \varepsilon}{\partial t}-\rho_{0} T \frac{\partial^{2} A}{\partial T^{2}} \frac{\partial T}{\partial t}=0
$$

By defining the specific heat as follows:

$$
c_{e}=-T \frac{\partial^{2} A}{\partial T^{2}}
$$

Equation (28) can then be re-written as:

$$
\rho_{0} c_{e} \frac{\partial T}{\partial t}=T \cdot \frac{\partial \sigma}{\partial T} \cdot \frac{\partial \varepsilon}{\partial t}
$$


It should be noted that a similar derivation for Equations (15) to (30) can be found in Wong, et al. ${ }^{35}$ and Bert and $\mathrm{Fu}^{20}$ when they investigated the thermoelastic parameter and thermal buckling taking into account the effect of stress on the coefficient of thermal expansion.

Now, assuming linear elastic behaviour, i.e. using Hooke's law:

$$
\sigma=E . \varepsilon
$$

where, $\sigma$ is the mechanical stress in the system, $E$ is Young's modulus and $\varepsilon$ is the strain of the material. It should be noted that the strain and Young's modulus in Equation (31) are functions of temperature.

By differentiating the stress of Hooke's law with respect to temperature, we obtain:

$$
\frac{\partial \sigma}{\partial T}=\frac{\partial}{\partial T}(E \varepsilon)=E \cdot \frac{\partial \varepsilon}{\partial T}+\varepsilon \cdot \frac{\partial E}{\partial T}
$$

Substituting the strain $\varepsilon$ of $\sigma / E$ from Hooke's law into Equation (32) yields:

$$
\frac{\partial \sigma}{\partial T}=E \cdot \frac{\partial \varepsilon}{\partial T}+\frac{\sigma}{E} \cdot \frac{\partial E}{\partial T}
$$

which can be re-arranged to give:

$$
\frac{\partial \sigma}{\partial T}=-E\left(-\frac{\partial \varepsilon}{\partial T}-\frac{\sigma}{E^{2}} \cdot \frac{\partial E}{\partial T}\right)
$$

By substituting Equation (34) into Equation (30), we obtain:

$$
\rho_{0} c_{e} \frac{\partial T}{\partial t}=-E T\left(-\frac{\partial \varepsilon}{\partial T}-\frac{\sigma}{E^{2}} \cdot \frac{\partial E}{\partial T}\right) \cdot \frac{\partial \varepsilon}{\partial t}
$$

For instances of stress being equal to zero, but $\partial \varepsilon / \partial T$ not necessarily equal to zero, the conservation of energy can be rewritten as:

$$
\rho_{0} c_{e} \frac{\partial T}{\partial t}=-E T\left(-\frac{\partial \varepsilon}{\partial T}\right) \cdot \frac{\partial \varepsilon}{\partial t}
$$

Equation (36) allows to define the free linear expansion coefficient $\left(\alpha_{0}\right)$ as:

$$
\alpha_{0}=-\frac{\partial \varepsilon}{\partial T}
$$

Combining this with Equation (35) while introducing a stress-dependent thermal expansion coefficient $\alpha_{\sigma}$ and the total thermal expansion coefficient $\alpha$, we have:

$$
\begin{gathered}
\rho_{0} c_{e} \frac{\partial T}{\partial t}=-E T\left(\alpha_{0}-\frac{\sigma}{E^{2}} \cdot \frac{\partial E}{\partial T}\right) \cdot \frac{\partial \varepsilon}{\partial t} \\
\alpha_{\sigma}=\frac{\sigma}{E^{2}} \frac{\partial E}{\partial T} \\
\alpha=\alpha_{0}-\alpha_{\sigma}=\alpha_{0}-\frac{\sigma}{E^{2}} \frac{\partial E}{\partial T} \\
\rho_{0} c_{e} \frac{\partial T}{\partial t}=-\alpha E T \frac{\partial \varepsilon}{\partial t}
\end{gathered}
$$


Accordingly, it can be concluded that the thermal expansion coefficient of a material subject to combined heating and loading condition is the summation of its free linear expansion coefficient and a stress-dependent expansion coefficient. The effects of stress on thermal expansion coefficient of material have thus been explicitly accounted for in Equation (40), which has been derived on the basis of only fundamental thermodynamics and continuum mechanics laws - with no mathematical fits to experimental data required.

Recognizing the dependency of all the parameters on temperature, Equation (3) can now be fully rewritten as:

$$
\varepsilon_{t o t}=\frac{\sigma(T)}{E(T)}+\alpha \cdot \Delta T
$$

where $\alpha$ is the total thermal expansion coefficient, comprising the free linear thermal expansion coefficient $\left(\alpha_{0}\right)$ and a stress-dependent thermal expansion coefficient $\left(\alpha_{\sigma}\right)$ as per Equation (40).

Equation (42) enables to effectively and rationally account for the combined effects of stress and temperature increase. The following section aims to highlight such capability of the model, with a focus on concrete - the most commonly used construction material. It should also be noted that the above relationships, Equation (42) included, are valid for a wide range of materials, as long as the associated assumptions made during derivation of the Equations are satisfied. It should also be noted that such assumptions are essentially consistent with typical testing regimes used for establishing constitutive model parameters for solid materials.

\section{Model verification}

We chose the original test data on concrete conducted by Anderberg and Thelandersson 10 and some available LITS correlations ${ }^{10,27,28}$ to verify the improved constitutive model. Two types of test data are used for this verification, including (i) the total strain recorded while maintaining constant stress during heating of the samples, and (ii) the total reaction force recorded while maintaining zero total strain during sample heating.

\section{Young's modulus at elevated temperatures}

The instantaneous Young's modulus is used in this model. This value is determined by using the unstressed test in which the sample is heated to target temperature, then maintained at the target temperature for 2-3 hours to achieve an essentially stable condition within samples. The sample is then loaded until failure to determine the stress-strain curve and Young's modulus value. To simplify the comparison, a simple Young's modulus model of concrete found in the literature ${ }^{36}$ is used in subsequent comparisons:

$$
E(T)=\frac{800-T}{740} \cdot E_{0} ; \text { for } 60^{\circ} \mathrm{C} \leq T \leq 800^{\circ} \mathrm{C}
$$

where $E_{0}$ is Young's modulus of concrete at ambient temperature.

Accordingly, the rate of change of Young's modulus respect to temperature is: 


$$
\frac{\partial E}{\partial T}=\frac{-E_{0}}{740}
$$

\section{Total strain correction}

As discussed in the previous section, the total strain of the concrete sample should take into account the step of applying a compressive load at ambient condition before heating, which creates an initial strain in concrete. In tests where the measured total strain is tared to zero (from a value caused by loading) shortly prior to heating, the following correction needs to be made to obtain the true total strain:

$$
\begin{gathered}
\varepsilon_{\text {tot }}\left(T_{a m b}, \sigma_{0}\right)=\frac{\sigma_{0}}{E_{0}} \\
\varepsilon_{\text {tot }}(\text { true })=\varepsilon_{\text {tot }}(\text { measured })-\frac{\sigma_{0}}{E_{0}}
\end{gathered}
$$

\section{Results and discussion}

To assess the predictive capability of the proposed model, its predicted values for the total strain and for the restraint stress are plotted in Figure 2 Error! Reference source not found.and Figure 3 respectively. The predictions are presented with the corresponding experimental data (Khoury, et al. ${ }^{12}$ and Anderberg and Thelandersson ${ }^{10}$ ) as well as the estimations obtained using available correlations ${ }^{10,27,28}$.

It can be clearly seen from Figures 2 and 3 that:

- Values of the total strain given by proposed model agree well with corresponding experimental data for all four stress levels reported (Figure 2). Such good agreement is observed for the entire range of temperatures tested, including those above $500^{\circ} \mathrm{C}$.

- The proposed model captures very well both the trend and magnitude of the restraint stress throughout the heating process (Figure 3 ). In contrast, the Anderberg's correlation gives less accurate predictions for temperatures above $200^{\circ} \mathrm{C}$, even though the correlation was developed on the basis of such test data.

Furthermore, major features characterizing the coupled effects of stress and temperature on total strain, which have been experimentally observed and reported as LITS in previous studies $^{1,7}$, can be logically explained or captured by the proposed model. To illustrate:

- The LITS developed during first heating is observed mostly irrecoverable on cooling: This can be explained by the negligible contribution of the stress-dependent thermal expansion coefficient $\alpha_{\sigma}$ to the total strain in Equation (42), resulted from the very small $\partial E / \partial T$ during cooling.

- Similarly, $\partial E / \partial T$ is also negligibly small during subsequent heating up to temperature levels lower than the maximum temperature reached during first heating. This helps explain the observed inappreciable additional LITS during such subsequent heating. 
- However, if temperature levels during subsequent heating are considerably higher than the maximum temperature during first heating, $\partial E / \partial T$ becomes significant; and hence, appreciable LITS should be expected.

The above statements are in good agreement with test data. The ability of the proposed model to capture and explain experimentally-observed features of the LITS component clearly demonstrates its strong predictive capability.

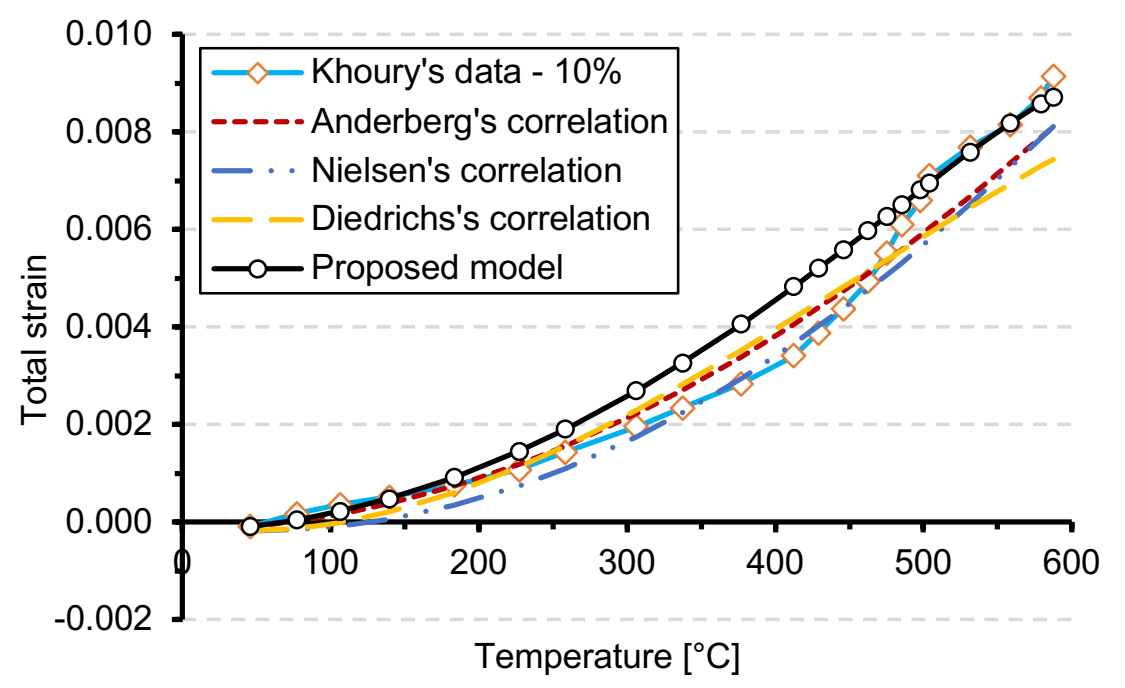

(a) For applied stress being $10 \%$ of ambient compressive strength.

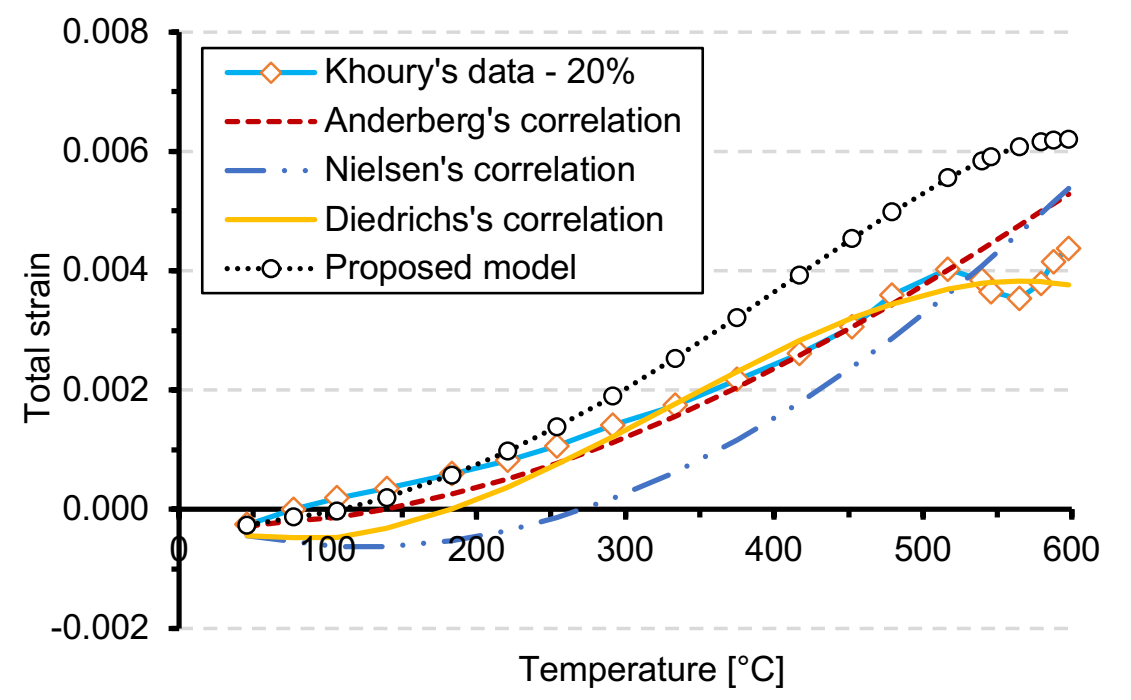

(b) For applied stress being $20 \%$ of ambient compressive strength. 


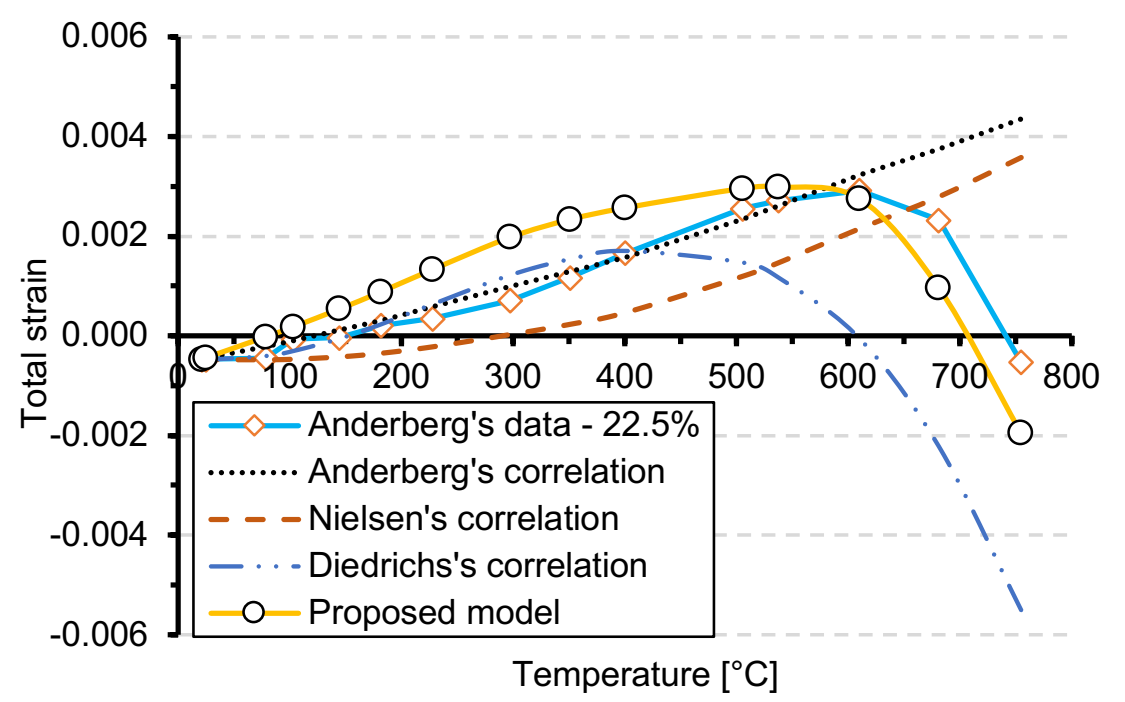

(c) For applied stress being $22.5 \%$ of ambient compressive strength.

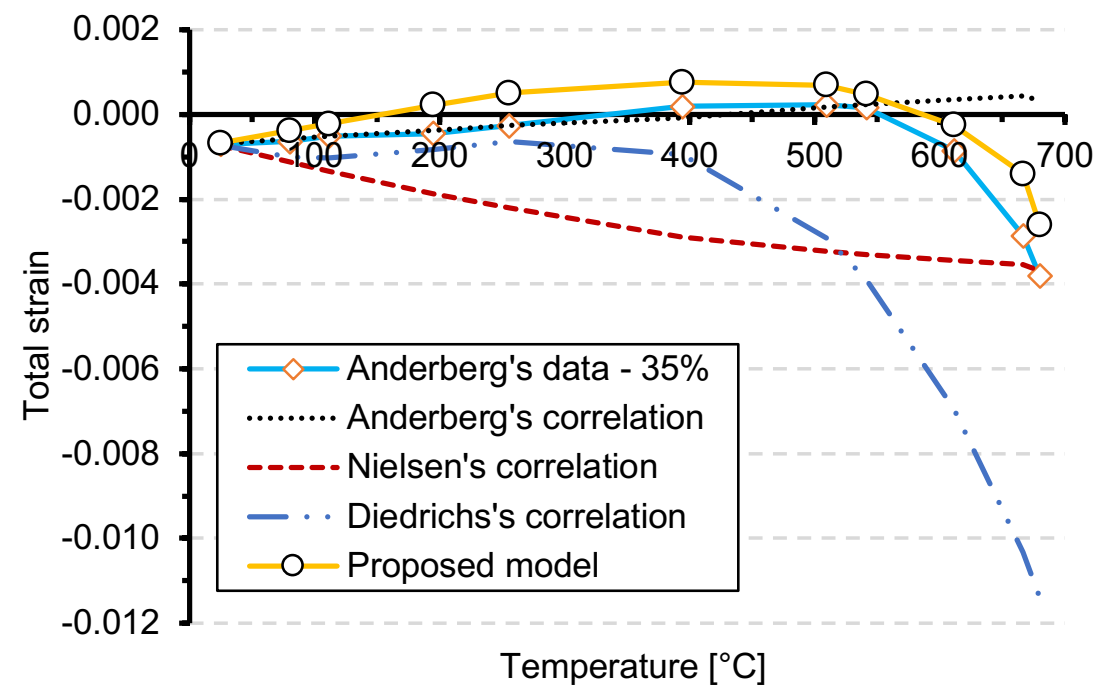

(d) For applied stress being $35 \%$ of ambient compressive strength.

Figure 2. Comparison between experimental (Khoury, et al. ${ }^{12}$ and Anderberg and Thelandersson ${ }^{10}$ ) and predicted values of the total strain of concrete at elevated temperatures under different stress levels. 


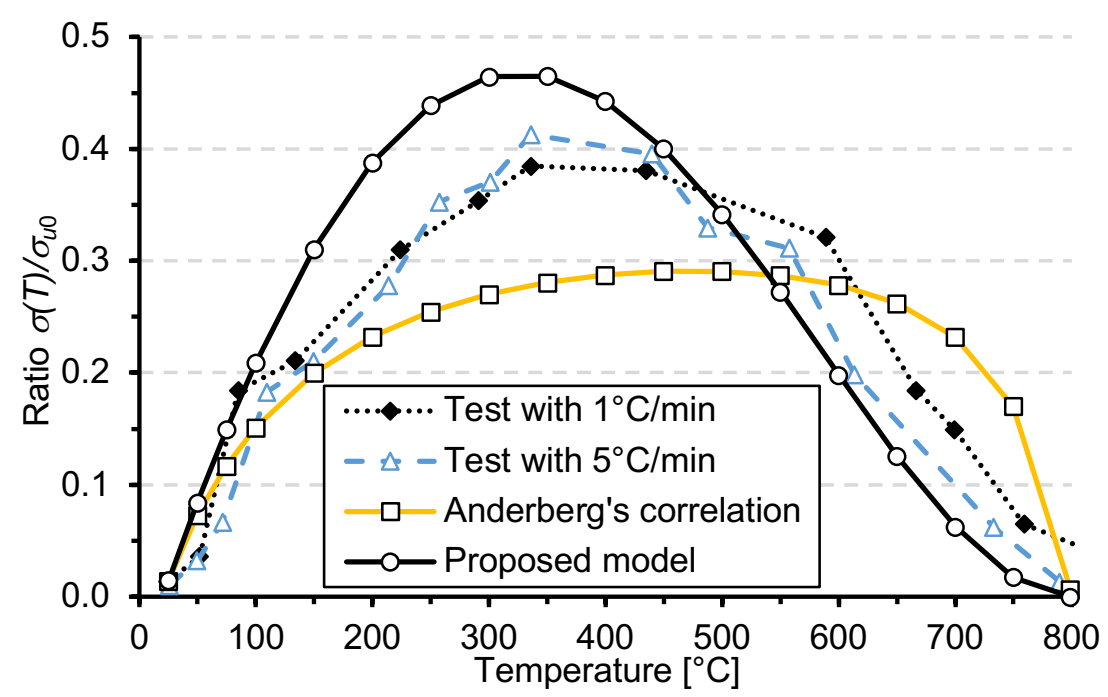

Figure 3. Comparison between experimental (Anderberg and Thelandersson ${ }^{10}$ ) and predicted values of restraint stress of heated concrete samples.

\section{Simple method for establishing parameters of the derived model}

Equation (42) can be re-written as:

$$
\varepsilon_{t o t}=\frac{\sigma(T)}{E(T)}+\left(\alpha_{0}-\frac{\sigma(T)}{E(T)^{2}} \frac{\partial E}{\partial T}\right) \Delta T
$$

On the basis of Equation (47), prior knowledge of $E(T)$ and $\partial E / \partial T$ is required to quantify the coupling effects. However, such knowledge may not be available for the material under consideration: Extensive testing is then required to collect needed data. Alternatively, assumptions on $E(T)$ and $\partial E / \partial T$ can be made; however, such assumptions necessarily introduce uncertainties that may not be easily quantifiable.

Now, re-arranging Equation (47):

$$
\varepsilon_{t o t}=\sigma(T)\left(\frac{1}{E(T)}-\frac{1}{E(T)^{2}} \frac{\partial E}{\partial T} \Delta T\right)+\alpha_{0} \Delta T
$$

By defining an effective Young's modulus of elasticity as $E_{\text {eff }}(T)$, where:

$$
\frac{1}{E_{e f f}(T)}=\frac{1}{E(T)}-\frac{1}{E(T)^{2}} \frac{\partial E}{\partial T} \Delta T
$$

Equation (48) becomes:

$$
\varepsilon_{t o t}=\frac{\sigma(T)}{E_{e f f}(T)}+\alpha_{0} \Delta T
$$

Equation (50) allows to conveniently determine parameters of the derived rational model (including $E_{\text {eff }}(T)$ ) using existing and widely-available tests. This aspect is critically significant in enabling the wide adoption of the model in engineering applications, thereby realising the potential impacts of fundamental science. 
The following scenarios are to further illustrate the simple determination of the model parameters:

a. If the sample is loaded to target stress at ambient temperature, Equation (48) can be simplified as indicated in Equation (50) and the constitutive model of the sample will be Hooke's law:

$$
\varepsilon_{\text {tot }}=\frac{\sigma_{0}}{E_{0}}+0=\frac{\sigma_{0}}{E_{0}}
$$

where, $\sigma_{0}$ is compressive stress at ambient temperature

o This test allows to obtain the Young's modulus of concrete at ambient temperature $E_{0}$.

b. If the sample is heated to the target temperature without any restraint condition, the stress in the sample will be zero. Equation (50) then becomes:

$$
\varepsilon_{t o t}(T)=\frac{0}{E(T)}+\alpha \cdot \Delta T=\alpha_{0} \cdot \Delta T
$$

- If the sample is heated in this case, the total strain is the free thermal expansion strain without any effects of stress on the thermal expansion coefficient.

- This test allows to obtain the free linear thermal expansion coefficient at temperature $T\left(\alpha_{0}\right)$

c. If the sample is heated to the target temperature with no restraint condition, then loaded to failure at such target temperature, the total strain of sample becomes:

$$
\varepsilon_{\text {tot }}(T)=\alpha_{0} . \Delta T+\varepsilon_{\sigma}
$$

where $\varepsilon_{\sigma}=\sigma / E(T)$.

- Therefore, this test allows to obtain the Young's modulus of concrete $E(T)$ and the stress-strain curve for concrete at a specific target temperature.

d. If the sample is loaded to target stress $\sigma_{0}$ and then heated to target temperature $(T)$ with the stress unchanged, the constitutive model will be:

$\circ$ Before heating:

$$
\varepsilon_{t o t}\left(T_{a m b}\right)=\frac{\sigma_{0}}{E_{0}}
$$

- After being heated to target temperature $(T)$ with stress $\left(\sigma_{0}\right)$ unchanged:

$$
\varepsilon_{t o t}(T)=\frac{\sigma_{0}}{E_{\text {eff }}(T)}+\alpha_{0} \Delta T
$$

- This test allows to determine the effective Young's modulus $E_{\text {eff }}(T)$ if the free thermal expansion coefficient $\left(\alpha_{0}\right)$ at temperature $T$ is known and the total strain $\varepsilon_{\text {tot }}$ is measured during testing.

e. If the total strain of the sample is fixed at zero while the sample is heated to a target temperature, the stress in the sample increases due to the expansion as follows:

○ Before heating: $\varepsilon_{\text {tot }}=0 ; \sigma=0$

○ After heating: $\varepsilon_{\text {tot }}=0 ; \sigma(T) \neq 0$

$$
\varepsilon_{\text {tot }}=\frac{\sigma(T)}{E_{e f f}(T)}+\alpha_{0} \Delta T=0
$$


- This test allows to determine the effective Young's modulus $E_{\text {eff }}(T)$ if the free thermal expansion coefficient $\left(\alpha_{0}\right)$ at temperature $\mathrm{T}$ is known and the restraint stress $\sigma(T)$ is captured during testing .

f. If the sample is loaded to a target stress and then heated to a target temperature while maintaining the total strain constant then the increased stress in the sample is given by:

○ Before heating: $\varepsilon_{t o t}=$ constant; $\sigma_{0} \neq 0$

$$
\varepsilon_{\text {tot }}=\frac{\sigma_{0}}{E_{0}}=\text { constant }
$$

- After heating: $\varepsilon_{\text {tot }}=$ constant; $\sigma(T) \neq \sigma_{0} \neq 0$

$\varepsilon_{\text {tot }}(T, \sigma)=\frac{\sigma(T)}{E_{\text {eff }}(T)}+\alpha_{0} \Delta T=\frac{\sigma_{0}}{E_{0}}=$ constant

- This test allows to determine or calibrate the coupling effect of stress and temperature increase.

\section{Summary and Conclusions}

In this paper, the need to account for the coupled effects of stress and temperature increase in assessing the performance of solid materials is first emphasized. It is then clearly highlighted that such effects are currently accounted for through the introduction of empirical correlations principally developed by best-fitting to limited test data. Accordingly, the applicability of existing correlations to scenarios different from those tested remains questionable and their reliability hinges on the reliability of corresponding test data.

Using fundamental thermodynamics and continuum mechanics laws, an equation showing the effects of stress on thermal expansion coefficient is then derived. On that basis, a new constitutive model for the total strain of solid materials under simultaneous loading and heating is proposed. The newly-proposed model is shown to have great predictive capability: (i) Its predictions agree well with available corresponding test data; and (ii) Key features of the coupling effects of stress and temperature increase on total strain, as experimentally observed in reported studies, can be logically explained by the model. The proposed model thus allows to reliably quantify such coupled effects of stress and temperature increase, thereby contributing to a more rational and reliable analysis and design of structures. Equally importantly, a simple method to conveniently determine parameters of the derived rational model is also presented, enabling the wide adoption of the model in engineering applications. Having been rationally developed from first principles, the proposed model should be applicable to a wide range of solid materials.

\section{Acknowledgment}

The financial support of Australian Research Council through LP140100504 and DP150102354 grants is gratefully acknowledged. First author also thanks to the financial support from the supervisors to conduct a short-term visiting at Department of Fire Protection Engineering, the University of Maryland, the USA. 


\section{References}

1 Torelli, G., Mandal, P., Gillie, M. \& Tran, V.-X. Concrete strains under transient thermal conditions: A state-of-the-art review. Engineering Structures 127, 172-188, doi:10.1016/j.engstruct.2016.08.021 (2016).

2 Vecchio, F. J., Agostino, N. \& Angelakos, B. Reinforced concrete slabs subjected to thermal loads. Canadian Journal of Civil Engineering 20, 741-753, doi:10.1139/193099 (1993).

3 Thelandersson, S. Modeling of combined thermal and mechanical action in concrete. Journal of Engineering Mechanics 113, 893-906, doi:10.1061/(ASCE)07339399(1987)113:6(893) (1987).

4 Rosenfield, A. R. \& Averbach, B. L. Effect of stress on the expansion coefficient. Journal of Applied Physics 27, 154-156, doi:10.1063/1.1722325 (1956).

5 Bert, C. W. \& Fu, C. Implications of stress dependency of the thermal expansion coefficient on thermal buckling. Journal of Pressure Vessel Technology 114, 189192, doi:10.1115/1.2929028 (1992).

6 Anderberg, Y. \& Thelandersson, S. Stress and deformation characteristics of concrete at high temperatures. 2. Experimental investigation and material behaviour model. (Bulletin of Division of Structural Mechanics and Concrete Construction, Bulletin 54; Vol. Bulletin 54). (Lund Institute of Technology. Division of Structural Mechanics and Concrete Construction, 1976).

7 Khoury, G. A., Grainger, B. N. \& Sullivan, P. J. E. Transient thermal strain of concrete: literature review, conditions within specimen and behaviour of individual constituents. Magazine of Concrete Research 37, 131-144, doi:10.1680/macr.1985.37.132.131 (1985).

8 Hansen, T. C. \& Eriksson, L. Temperature change effect on behavior of cement paste, mortar, and concrete under load. Journal of the American Concrete Institute 63, 489-504 (1966).

9 Bažant, Z. P. \& Chern, J.-C. Stress-Induced Thermal and Shrinkage Strains in Concrete. Journal of Engineering Mechanics 113, 1493-1511, doi:10.1061/(ASCE)0733-9399(1987)113:10(1493) (1987).

10 Anderberg, Y. \& Thelandersson, S. Stress and deformation characteristics of concrete at high temperatures: 2 . Experimental investigation and material behavior model. Bulletin 54, 85 (1976).

11 Mindeguia, J. C., Hager, I., Pimienta, P., Carre, H. \& Borderie, C. L. Parametrical study of transient thermal strain of ordinary and high performance concrete. Cement and Concrete Research 48, 40-52 (2013).

12 Khoury, G. A., Grainger, B. N. \& Sullivan, P. J. E. Strain of concrete during first heating to $600^{\circ} \mathrm{C}$ under load. Magazine of Concrete Research 37, 195-215, doi:10.1680/macr.1985.37.133.195 (1985).

13 Terro, M. J. Numerical modeling of the behavior of concrete structures in fire. ACI Struct J 1998;95:183-93. ACl Structural Journal 95, 183-193 (1998).

14 Anderberg, Y. \& Thelandersson, S. Stress and deformation characteristics of concrete at high temperatures: 2 . Experimental investigation and material behavior model. (University of Lund, Sweden, 1976).

15 Sadd, M. H. Elasticity - Theory, Applications, and Numerics (2nd Edition). (Elsevier, 2009). 
17 Hetnarski, R. B., Eslami, M. R. \& Gladwell, G. M. L. Thermal stresses: Advanced theory and applications. (Dordrecht: Springer Netherlands, 2009).

18 Rosenfield, A. R. \& Averbach, B. L. Effect of stress on the thermal expansion coefficient. Journal of Applied Physics 27 (1956).

19 Maugin, G. A. Continuum mechanics through the eighteenth and nineteenth centuries. (Cham, Switzerland Heidelberg, Germany : Springer International Publishing, 2014).

20 Bert, C. W. \& Fu, C. Implications of stress dependency of thermal expansion coefficient on thermal buckling. Journal of Pressure Vessel Technology, Transactions of the ASME 114, 189-192 (1992).

21 Anderberg, Y. Modelling steel behaviour. Fire Safety Journal 13, 17-26, doi:https://doi.org/10.1016/0379-7112(88)90029-X (1988).

22 Knobloch, M., Pauli, J. \& Fontana, M. Influence of the strain-rate on the mechanical properties of mild carbon steel at elevated temperatures. Materials \& Design 49, 553-565, doi:https://doi.org/10.1016/j.matdes.2013.01.021 (2013).

23 Lee, J. Elevated-temperature properties of ASTM A992 steel for structural-fire engineering analysis Doctor of Philosophy thesis, The University of Texas at Austin, (2012).

24 Mindeguia, J.-C., Hager, I., Pimienta, P., Carré, H. I. n. \& Borderie, C. L. Parametrical study of transient thermal strain of ordinary and high performance concrete. Cement and Concrete Research 48, 40-52 (2013).

25 European Standard. in EN 1992-1-2 (2004): Eurocode 2: Design of concrete structures - Part 1-2: General rules - Structural fire design (European committee for Standardization, Brussiles, Belgium, 2004).

26 Gernay T \& Franssen JM. Consideration of transient creep in the Eurocode constitutive model for concrete in the fire situation. Proceedings of the sixth international conference structures in fire, 784-791 (2010).

27 Nielsen, C. V., Pearce, C. I. \& Bicanic, N. Theoretical model of high temperature effects on uniaxial concrete member under elastic restraint. Magazine of Concrete Research 54, 239-249, doi:10.1680/macr.2002.54.4.239 (2002).

28 Diederichs, U. Modelle zur Beschreibung der Betonverformung bei instantion \{ä\}ren Temperaturen. Abschlu\{ß\}kolloquium-Bauwerke Unter Brand, 25-34 (1987).

29 Li, L. \& Purkiss, J. Stress-strain constitutive equations of concrete material at elevated temperatures. Fire Safety Journal 40, 669-686 (2005).

30 Khoury, G. A., Sullivan, P. J. E. \& Grainger, B. N. Transient thermal strain of concrete: literature review, conditions within specimen and behaviour of individual constituents. Magazine of Concrete Research 37, 131-144 (1985).

31 Gernay, T. \& Franssen, J. M. A formulation of the Eurocode 2 concrete model at elevated temperature that includes an explicit term for transient creep. Fire Safety Journal 51, 1-9, doi:10.1016/j.firesaf.2012.02.001 (2012).

32 European Standard. in ENV 1992-1-2:1995: Eurocode 2: Design of concrete structures - General rules - Structural fire design (European Committee for Standardization (CEN), Brussels, 1995).

33 Schneider, U. Behaviour of concrete at high temperature. Deutscher Ausschuss fur Stahlbeton, Berlin (1982). 
34 Gernay, T. Effect of Transient Creep Strain Model on the Behavior of Concrete Columns Subjected to Heating and Cooling. Fire Technology 48, 313-329, doi:10.1007/s10694-011-0222-0 (2012).

35 Wong, A. K., Jones, R. \& Sparrow, J. G. Thermoelastic constant or thermoelastic parameter? Journal of Phys. Chem. Solids 48, 749-753 (1987).

36 Aslani, F. \& Bastami, M. Constitutive relationships for normal-and high-strength concrete at elevated temperatures. ACI Mater. J. 108, 355-364 (2011). 